Oh, W., Yao, A. C., and Hanson, J. S. (1972). The effects of phototherapy on peripheral blood flow and insensible water loss in newborn infants (abstract). Clinical Research, 20, 283.

Oh, W., Yao, A. C., Hanson, J. S., and Lind, J. (1973). Peripheral circulatory response to phototherapy in newborn infants. Acta paediatrica Scandinavica, 62, 49-54.

Wu, P. Y. K., and Berdahl, M. (1974). Irradiance in incubators under phototherapy lamps. Journal of Pediatrics, 84, 754-755.

Wu, P. Y. K., Wong, W. H., Hodgman, J. E., and Levan, N. (1974). Changes in blood flow in the skin and muscle with phototherapy. Pediatric Research, 8, 257-262.

AleXander Habel and Ian Swann

University of Edinburgh, and Simpson Memorial Maternity Pavilion, and Elsie Inglis Maternity Hospital, Edinburgh

Correspondence to Dr A. Habel, West Middlesex Hospital, Isleworth, Middlesex TW7 6AF.

\section{Slow growth of an untreated Wilms's tumour in the adolescent}

\section{Case history}

A 16-year-old white girl presented symptomatically in May 1976 with left upper quadrant discomfort and fatigue. She had been seen by her family physician, found to be mildly anaemic with $\mathrm{Hb}$ $10.5 \mathrm{~g} / \mathrm{dl}$, and placed on iron treatment. In August she lost $8.63 \mathrm{~kg}$ during a 2-week period and developed anorexia, night sweats, and dyspnoea. She was admitted to a local hospital and $x$-rays and urograms taken in September showed a large renal mass on the left side (Fig. 1), and the chest

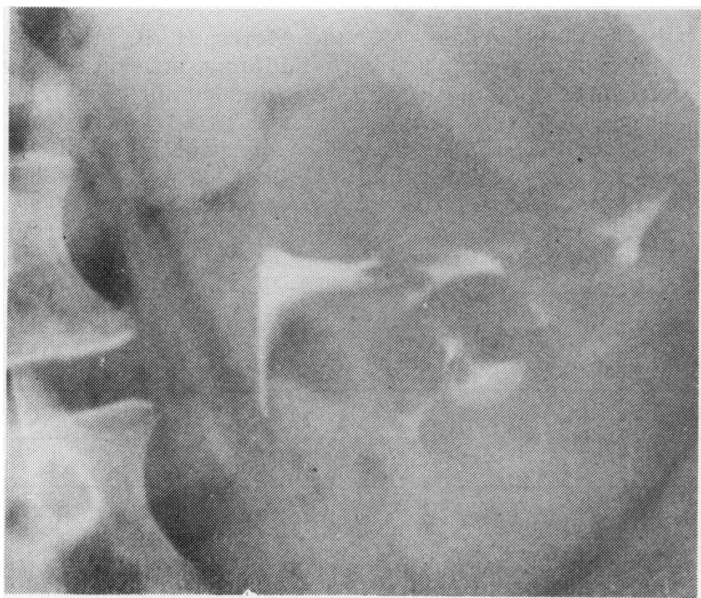

Fig. 1 1976. Huge intrarenal mass with distortion and displacement of calyces. $x$-ray revealed pulmonary metastases. She subsequently developed increasing distress due to a left pleural effusion and was transferred to Vancouver General Hospital.

History revealed recurrent bouts of cystitis between ages 5 and 11 years, during which time she had received treatment with antibiotics. At age 11 she had seen a urologist who carried out a urogram. Cystoscopy and urethral dilatation had also been undertaken. Figs 2 and $3, x$-rays taken in February 1972, show an intrarenal mass consistent with Wilms's tumour. Subsequently she had an aching flank on the left side believed related to chronic urinary tract infection.

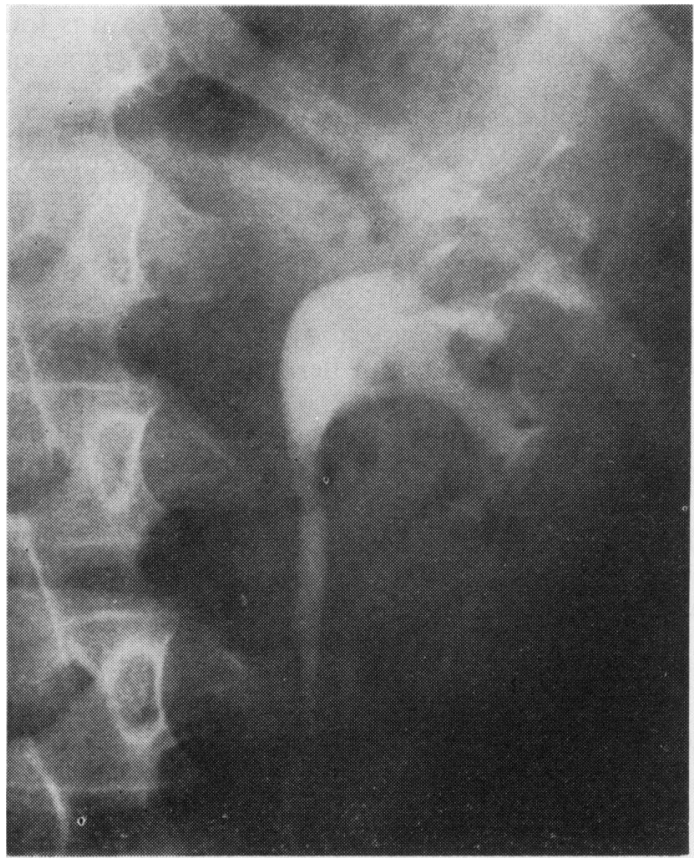

Fig. 2 1972. Depression and stretching of superior calyx. Evidence of space occupying lesion in superior pole.

On presentation to Vancouver General Hospital with metastatic Wilms's tumour in 1976 she underwent drainage of the pleural effusion and was treated initially with vincristine and then with actinomycinD. This was followed by irradiation to the thorax. There was regression of the pulmonary metastases and abdominal mass, and surgery was then performed to remove the renal tumour. Splenectomy was also done at time of surgery due to attachment of the mass. The tumour excision was not complete. Histology confirmed the diagnosis of Wilms's 


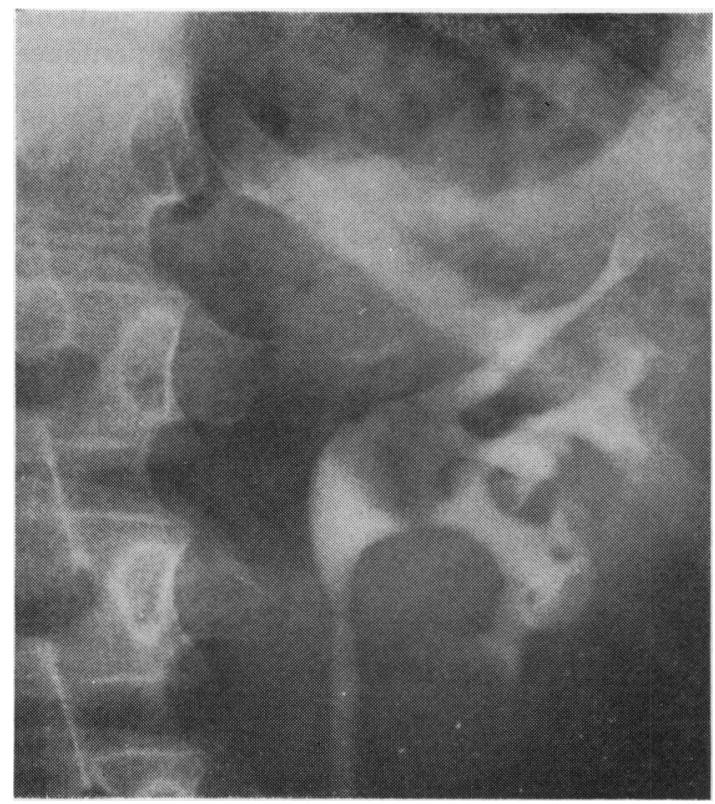

Fig. 3 1972. Depression and stretching of superior calyx. Evidence of space occupying lesion in superior pole.

tumour of sarcomatous type as seen by two independent pathologists. No congenital abnormalities of the renal system were noted at operation. She received further irradiation to the whole of the abdomen and treatment with vincristine, actinomycinD, and adriamycin. Currently she is clinically well with continuing radiological evidence of pulmonary disease, and remains on this regimen.

\section{Discussion}

The natural history of Wilms's tumour and its growth have recently been altered by new multimodality treatment. It is usually regarded as a rapidly growing tumour and many hospitals approach initial surgical management as a semiemergency.

Estimates of doubling time in human Wilms's cells show a median potential cell doubling time of 188 hours (7.8/day) (Aherne and Buck, 1971). Radiographic measurements of the growth of pulmonary metastases showed a median value of 20 days for overall tumour volume doubling time (Holland, 1976). There are records of clinically observed rapid growth of the primary renal tumour with variable time intervals between normal excretory urograms and repeat studies for abdominal masses. It was not possible to give a definite age and tumour growth correlation in these reports (Grossman, 1976). We show here an interval of nearly 4 years of slow growth before the clinical metastatic presentation. There therefore may be a varied natural growth pattern of Wilms's tumour influenced by age, histology, or factors yet unknown. Metastatic disease in long-term survivors is being reported, also indicating nongrowth or a slow growth period of micrometastases that later begin to proliferate (Cassady et al., 1973). This varied growth rate was suggested by Collins to be related with age, as his classic formula for period at risk indicates (Collins, 1955, 1958). Prognosis is known to be worse in the older patient (D'Angio et al., 1976). One can hypothesise whether this is due to a slower or smaller growth fraction of tumour and is therefore less sensitive to cycle specific agents. Alternatively it may be due to the more anaplastic or sarcomatous type of histology associated with a poorer prognosis (Perez et al., 1973) and possibly occurring more frequently in the older patient. Further study into the growth pattern of Wilms's tumours is necessary and of the relationship of these tumours with age and histology.

\section{Summary}

Retrospective radiological documentation of a growing Wilms's tumour is rare. Considered to be a relatively rapidly growing tumour, we present a case where there was adequate radiological evidence of a Wilms's tumour 4 years before the clinical metastatic presentation.

\section{References}

Aherne, W., and Buck, P. (1971). The potential cell population doubling time in neuroblastoma and nephroblastoma. British Journal of Cancer, 25, 691-696.

Cassady, J. R., Tefft, M., Filler, R. M., Jaffe, N., and Hellman, S. (1973). Considerations in the radiation therapy of Wilms' tumor. Cancer, 32, 598-608.

Collins, V. P. (1955). Wilms' tumor: its behaviour and prognosis. Journal of the Louisiana State Medical Society, 107, 474-480.

Collins, V. P. (1958). The treatment of Wilms' tumor. Cancer, 11, 89-94.

D'Angio, G. J., Evans, A. E., Breslow, N., Beckwith, B., Bishop, W., Feigl, P., Goodwin, W., Leape, L. L., Sinks, L. F., Sutow, W., Tefft, M., and Wolff, J. (1976). The treatment of Wilms' tumor-results of the National Wilms' Tumor Study. Cancer, 38, 633-646.

Grossman, H. (1976). Observing the growth of Wilms' tumor. Radiology, 121, 697-700.

Holland, P. (1976). Clinical and biochemical manifestations of Wilms' tumor. In Wilms' Tumor, p. 15. Edited by C. Pochedly and D. Miller. Wiley: New York. 
Perez, C. A., Kaiman, H. A., Keith, J., Mill, W. B., Vietti, T. J., and Powers, W. E. (1973). Treatment of Wilms' tumor and factors affecting prognosis. Cancer, 32, 609-617 .

Paul C. Rogers, Betty J. Wood, David F. Smith, AND J. M. TEASDALE

Departments of Paediatrics and Diagnostic Radiology, University of British Columbia, Vancouver, British Columbia, Canada V5Z 1 M9

Correspondence to Dr P. C. J. Rogers, Department of Paediatrics, University of British Columbia, 715 West 12th Avenue, Vancouver, British Columbia, Canada V5Z 1 M9.

\section{Thermometers and rectal perforations in the neonate}

Spontaneous perforation of the gastrointestinal tract in the neonate is rare. When it occurs it usually involves the stomach or the ileocaecal region. Perforation of the rectum in the neonate is usually caused by instruments being passed into it. Two patients with neonatal rectal perforations, probably caused by thermometers, have been admitted to the hospital during the last 6 months. Only one other case could be found from our records during the last 15 years.

\section{Case reports}

Case 1. A baby boy, birthweight $4.5 \mathrm{~kg}$, term, forceps delivery for delay in the 2nd stage of labour. The abdomen was noted to be mildly distended at birth. At age 7 hours, while the baby's temperature was being taken rectally, the thermometer snapped. The abdomen became more distended and an erect $x$-ray showed free gas in the peritoneal cavity. At laparotomy on the same day, there was copious peritoneal fluid and the small bowel was matted together with fibrin. There was a $4 \mathrm{~mm}$ perforation in the anterior rectal wall. The perforation was oversewn and a double barrel transverse colostomy performed. The baby's recovery was uneventful and the colostomy was closed 6 months later.

Case 2. A baby girl, birthweight $2.6 \mathrm{~kg}$, term, normal delivery. She was well until 4 days old when she suddenly became ill with circulatory collapse and marked abdominal distension. An erect $x$-ray of the abdomen showed free gas in the peritoneal cavity. After resuscitation, at laparotomy, there was gross faecal peritonitis. The rectum was contused for a distance of $2 \mathrm{~cm}$ above the peritoneal reflection and there were 2 perforations in the anterior wall of the rectum. The cause of this damage was unknown at the time of operation so the contused segment was excised, the rectum oversewn, and the sigmoid colon brought out as an end colostomy. Histological examination of the resected specimen confirmed the severe contusions and haemorrhage but the mucosa was found to be undamaged except for the perforations. The postoperative course was complicated by a wound dehiscence but was otherwise satisfactory and the patient is now awaiting a rectosigmoid anastamosis.

Case 3. A baby boy, birthweight $2.9 \mathrm{~kg}$, term, normal delivery. On the 2 nd day of life, having passed meconium and taken his feeds well, he began to vomit bile-stained material and the abdomen became distended. An erect $x$-ray of the abdomen showed free gas in the peritoneal cavity. He was resuscitated and at laparotomy there was a large amount of turbid fluid in the peritoneal cavity. After an extensive search a perforation, $5 \mathrm{~mm}$ in diameter, was found in the anterior rectal wall at the level of the peritoneal reflection. The perforation was oversewn and a loop sigmoid colostomy performed. He made an uneventful recovery and the colostomy was closed 6 weeks later.

\section{Discussion}

As with most previous reports it was not always easy to prove that a thermometer was the cause of the rectal perforation. In Case 1 the thermometer actually snapped inside the baby's rectum. Cases 2 and 3 had been transferred from different maternity units where it was normal practice to measure temperatures rectally. Although the actual incident causing the perforation could not be identified the lesions were characteristic. The presence of a small circumscribed perforation in the anterior rectal wall at the level of the peritoneal reflection, in a baby transferred from a hospital where the rectal thermometer is routinely used, is sufficient to implicate the procedure. A 4th baby has been admitted to this hospital with clinical and laparotomy findings similar to those reported above. Although the lesion appeared to be the result of instrumentation of the rectum, the baby had been born at home and no evidence of the insertion of a thermometer into the rectum could be obtained.

Neonatal rectal perforations have been observed after the passing of various types of instruments, such as catheters used in rectal washouts and barium 